\section{LA-7359-P}

Proposal

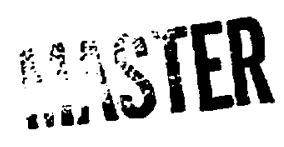

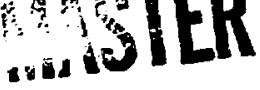

A Proposal for the Theoretical Investigation of the Relativistic Beam-Plasma Interaction with Application to the Proof-of-Principle

Electron Beam-Heated Linear Solenoidal Reactor

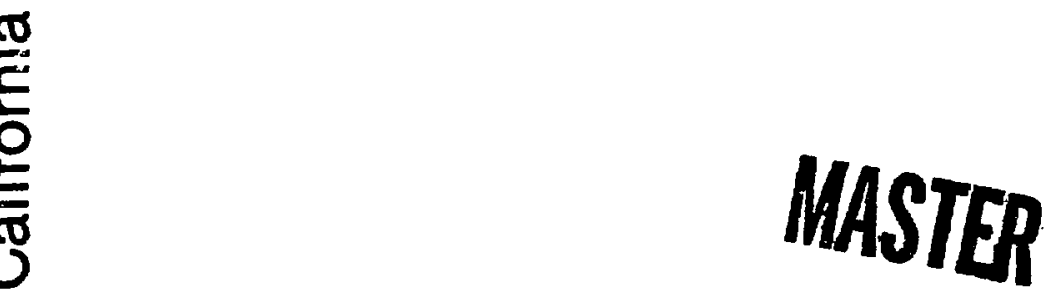

임

$\frac{7}{20}$ 
LA-7359-P

Proposal

Special Distribution

Issued: September 1978

\title{
A Proposal for the Theoretical Investigation of the Relativistic Beam-Plasma Interaction with Application to the Proof-of-Principle \\ Electron Beam-Heated Linear Solenoidal Reactor
}

\author{
Lester E. Thode
}

This report was prepared as an account of wotk
sponsored by the United States Government. Neithes the
United States nor the United States Department of
Energy, nor any of their employes, nos any of the is
cantractors, subconteacton, of their employees, makes
any wartanty, express of implied, $r$ assumes any legal
Liability or sesponsibility for the accuracy, completeness
or usefuiness of any information, apparatus, product or
process disclosed, or represents that its use would not
infringe privalefy owned rights.

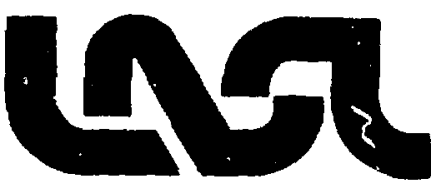


A PROPOSAL FOR THE THEORETICAL INVESTIGATION OF THE

RELATIVISTIC BEAM-PLASMA INTERACTION WITH APPLICATION TO THE

PROOF-OF-PRINCIPLE ELECTRON BEAM-HEATED LINEAR SOLENOIDAL REACTOR

by

Lester $E$. Thode

\begin{abstract}
A 36-month program to study the linear relativistic electron beam-plasma interaction is proposed. This program is part of a joint proposal between the Physics International Company (PI) and Los Alamos Scientific Laboratory (LASL) that combines the advancied electron beam generator technology at PI with the highly developed computer simulation technology at LASL. The proposed LASL program includes direct support for 1- and 3-m beam-plasma interaction experiments planned at PI and development of theory relevant for design of a $10-m$ proof-of-principle electron beam-driven linear solenoidal reactor.
\end{abstract}

\title{
I. INTRODUCTION
}

For the linear solenoidal reactor application, it is required that the intense relativistic electron beam convert its streaming energy into plasma thermal energy as it propagates through the solenoid, in an approximately uniform and controllable fashion. Depending upon the plasma density, magnetic field strength, and end loss reduccion, linear solenoid reactors are expected to be in the range of $300-500 \mathrm{~m}$ in length. ${ }^{l}$ For relativistic electrons this distance is considerably shorter than the classical deposition length. Thus. the energy transfer must be anomalous. Experiments to investigate the feasibility of using intense relativistic beams to heat a plasma have all reported anomalous beam coupling. ${ }^{2-7}$ The interpretation of the experiments with respect to the source of the anomalous deposition has varied, however. 
As currently understood, there are basically two mechanisms through which the beam's streaming energy can be converted to plasma thermal energy. First, the local beam momentum distribution function can be modified because of highfrequency wave generation. Plasma heating by this mechanism is associated with the two-stream and upper-hybrid instabilities, and can be characterized as relaxation heating. Second, plasma heasing caused by modification of the beam distribution function resulting from ar enhanced inductive electric-field drag is expected. This mechanism is generally referred to as resistive heating. As originally proposed, ${ }^{8}$ ion-acoustic turbulance resulting from the relative drift between the plasma electrons and ions vas to provide the anomalous conductivity necessary for resistive heating. However, ion-acourtic turbulence is short lived and cannot be relied upon to resistively heat a dense plasma to thermonuclear temperature. 1,9 Thus, for both heating mechanisms, it is the generation of the high-frequency wave spertrum that leads to the anomalous energy ioss.

For the linear solenoidal reactor, it is conventionally accepted that the coupling will be determined by the kinetic interaction. Here kinetic refers to the generation. of high-frequency waves by resonant or Landau growth. The kinetic heating approach is based upon a slow, and thus more controlled, heating rate over the entire beam pulse of a plasma column that extends the entire length of the reactor.

An alternative heating approach, which would be applicable to initially producing a shorter (fraction of the total reactor length) but slightly higher density and temperature plasma near the center of the reactor, appears possible. In this approach, the local energy deposition rate is very rapid but occurs for only a small fraction of the beam pulse. Specifically, the streaming instabilities are hydrodynamic in character but are stabilized locally after a time $\tau_{I}$ by a longitudinal density gradient. The density gradient is the result of additional ionization produced by the spatially dependent heating associated with the hydrociynamic interaction. Provided that the lifetime of the density gradient is longer than the beam pulse length, the beam will pass through previously heated plasma and interact with the next section of plasma. Continuing, the entire plasma column will be heated approximately uniformly at 40-70\% coupling efficiency. In addition, a simple relation exists between the beam pulse length $\tau$ and plasma column length $L=\left(\tau / \tau_{I}\right) L_{N}$, where $L_{N} \cong 2.0$ to $100 \mathrm{~cm}$ is the hydrodynamic deposition lengt.h in a magnetic containment device. For 
example, taking $\tau_{I}=10 \mathrm{~ns}$ and the beam pulse length equal to the lifetime of the ionization gradient $\tau_{G}=L_{N} / C_{S}, L=20$ to $100 \mathrm{n}$ for an ion-acoustic speed of $\mathrm{C}_{\mathrm{S}}=10^{8} \mathrm{~cm} / \mathrm{s}$.

In recent years, many theoretical calculations have been concerned with the relativistic electron beam-plasma interaction. The calculations can be split into two categories according to the type of interaction: i) hydrodynamic, ${ }^{10-19}$ in which the coupling efficiency is determined by particle trapping, and ii) kinetic, ${ }^{20-26}$ in which the interaction is generally assumed to be limited by nonlinear processes. The study of the nonlinear beam relaxation in the hydrodynamic regime has primarily relied on analytical and numerical models, through partial numerical and paricicle code simulation techniques. On the other hand, investigation of the kinetic interaction has been dominated by quasi-linear/weak turbulence salculations. Exceptions are recent: calculations concerning parametric coupling of the primary turbulence to low-frequency waves. 24-26

Calculations for the kinetic inceraction are based upon assumptions concerning the nonlinear steady state spectrum, generally linear theory, from which the beam relaxation length can be obtained. Unfortunately, results of the calculations vary considerably because of the uncertainty in the assumed nonlinear spectrum and dominant nonlinear mechanism. Recent two-dimensional particle simulations ${ }^{19}$ for continuous injection of a cold beam into a target plasma indicate that the steady state spectrum is not a priori determineo by linear theory considerations, even if the fastest growing mode is initially along the direction of beam propagation. We anticipate that this character will also be present in the kinetic interaction, especially if longitudinal modes are limited to low levels by nonlinear mechanisins.

From an experimental standpoint, ${ }^{2}$ over $85 \%$ of the beam-plasina interaction data were obtained in the quasi-hydrodynamic regime, that is, the interaction was not truly hydrodynamic or kinetic in character. As a result, the experimental characteristics of the kinetic heating regime are not well established.

Relative to the kinetic heating regine, the hydrodynamic heating regime is well understood. ${ }^{2,10-19}$ In fact, beam self-magnetic field ${ }^{19}$ and plasma longitudinal density gradients are the only effects for which quantitative mocieis have not been developed. The study of both these effects has been included in this proposal. 
From the above considerations, the main objective of the proposed theoretical study is to understand the dominant characteristics associated with the anomalous coupling in the kinetic regime. One of the fundamental considerations is to determine if a dominant heating mechanism exists. To a great extent, this determination will depend on whether the high-frequency turbulence is directly absorbed by plasma electrons or ions through nonlinear Landau damping $"$ or is converted into low-frequency turbulence ${ }^{24-26}$ that can lead to anomalous resistance. Based upon nonlinear calculations and simulations, this turbulence appears to depend on the dimensionality of the high-frequency spectrum, which ultimately is expected to be determined by the angle and energy spread of the local beam distribution function and external magnetic field strength.

This theoretical study is part of a joint proposal between the Physics International Company ${ }^{1}$ (PI) and Los Alamos Scientific Laboratory (LASL). Reporting and documentation will be in conjunction with PI.

\section{TECHNICAL APPROACH AND OVERVIEW}

The primary theoretical task is to obtain an accurate nonlinear spectrum and resultant beam energy loss associated with the streaming instabilities in the kinetic regime. To achieve this task, the analysis will be done at five levels of sophistication: $i$ ) development of accurate linear theory; $i i)$ development and implementation of oblique, multiwave partial numerical simulation; iii) analytical quasi-linear/weak turbulence calculations; iv) one-dimensional particle code simulation; and $v$ ) two-dimensional particle code simulation. The exact interplay between the various techniques is discussed in secs. III - $v$. A. Accurate Linear Theory

The dependence of the instabilities on the following parameters will be considered: $i)$ diode voltage, $i i)$ beam-to-plasma density ratio, $i i i$ ) external magnetic field strength, iv) beam scatter and energy spread, v) plasma temperature, and vi) electron-ion collision rate. Because of the theoretical difficulty associated with temperature effects, the main approach will be, by necessity, numerical, although anaiytical results will be obtained when possible. Based upon previous investigation, ${ }^{19,27}$ radial boundary conditions and radial beam or plasma gradients are not expected to modify the plasma heating interactions significantly for plasma densities above $10^{15} \mathrm{~cm}^{-3}$. For a homogeneo'is 
plasma, linear theory is obtained from the numerical solution of a transcendental equation.

Beam stability will be studied using a coupled linear differential equation code that includes finite radial boundaries and radial particle profiles.

B. Oblique, Multiwave Partial Numerical Simulation

This technique treats the plasma by its dielectric properties, whereas the beam trajectories are computed numerically. The advantage of this technique is that the nonlinear state is solved for on the beam time scale. Thus, a large parameter study can be carried out for approximately the same cost as a single particle code simulation. In the past, this technique has been carried out primarily for single-wave hydrodynamic trapping calculation: 10,15-16 However, it can be extended straightforwardly into the kinetic regime by going to a multiwave spectrum. ${ }^{28,29}$ The oblique approximation ${ }^{10}$ is a one-dimensional calculation for the wave spectrum propagating at sone angle witi respect to beam propagation, the result of which is the relative nonlinear strength of the two-dimensional spectrum. To obtain these caiculations, a very accurate knowledge of the unstable spectrum (linear theory) is required.

C. Quasi-Linear/Weak Turbulence Calculations

From the knowledge of the ronlinear state, appropriate analytical estimates can be achieved within the quasi-linear/weak turbulence approximation. This analysis includes a review of existing calculations with respect to their initial assumptions on the nonlinear spectrum. $20-26$

D. One-Dimensional Particle Simulations

The essential characteristics of the quasi-linear relaxation, as well as the nonlinear mechanisms, will be investigated in the one-dimensional approximation using the electromagnetic particle code REL. ${ }^{30}$ Such calculations will provide checks for the partial numerical simulation techriques and some proposed nonlinear processes. These spatial injection runs will aiso provide estimates for anomalous resistivity and evolved distribution furctions.

E. Two-Dimensional Particle Simulations

For homogeneaus plasma interactions with no finite boundary conditicns, a two-dimensional, rectangular, electromagnetic code WAVE ${ }^{31}$ wi i be used. $A$ new electromagnetic code CCUBE, ${ }^{32}$ which is two-dimensional in any separable, orthogonal cocrdinate system, will be used to study the spatial injection problem on a short system. The code has experimental diagnostics such as 
Faraday cups, calorimeters, diamagnetic loops, Rugowski loops, local field probes, and local current probes. 33 Estimates for anomalous resistivity and evolved distribution functions will be obtained that can be compared to the more restrictive one-dimensional results.

In addition, the code CCUBE will be used extensively to study diode characteristics, beam self-field effects, and adiabatic compression. All CCUBE simulations will be done in a cylindrical or spherical ccordinate system.

The results of the analytical and numerical calculations for the nonlinear spectrum and anomalous resistivity will yield local heating rates. In addition, this detailed knowledge will be incorporated into hybrid codes developed at PI and LASL to investigate radial beam diffusion and deposition on an experimental time and length scale.

\section{PROPOSED FIRST-YEAR PROGRAM}

During the first year of the program, the effort will be directed toward the isolation of realistic beam distribution functions and the development of accurate linear theory. To obtain insight into the form of realistic distribution functions, some two-dimensional simulations co study steady state injection, beam self-magnetic field effects, and adiabatic compression will be done. A. Task 1: Linear Theory - Scattered Beam

Accurate linear theory is required for i) comparing those analytical calculations 24-25 that consider the linear growth rate as a parameter with the PI experimental results, ii) obtaining estimates for quasi-linear relaxation lengths and aillowed nonlinear interactions, iii) implementing multiwave partial numerical simulation codes and quasi-linear calculations, and iv) designing and implementing one- and two-dimensional particle code simulations that are grid independert.

As a starting point, the linear theory associated with the scattered beam distribution function ${ }^{2,18}$ will be extended to include an external magnetic field and plasma temperature. The beam and plasma will be assumed to be homogeneous and infinite in extent. The analysis will be performed including electromagnetic contributions and within tîie electrostatic limit. Based upon previous calculations, ${ }^{34}$ electromagnetic contributions are expected to modify only the most unstable regions of the spectrum for $w_{c} / w_{p} \geq 1$, where $w_{c}=e B_{z} / m c$ is the plasma cyclotron frequency associated with the external magnetic field $B_{z}$. 
The expected results from the analysis are both the temporal and spatial growth rate, frequency, spectrum width, phase velocity, and group velocity. It is also anticipated that analytic expressions can be obtained in the true kinetic regime. Criteria for the character of the streaming instabilities (hydrodynamic, quasi-hydrodynamic, or kinetic) are also expected. Finally, initial insight into the possible dimensionality of the steady state spectrum might be forthcoming. Fir the steady state condition, the strongest nonlinear modes are not, however, a priori associated with the fastest growing modes. B. Task 2: Estimates for Allowed Nonlinear Interactions - Scattered Beam

There have been a number of interactions assumed to be important in determining the steady state spectrum: i) resonant waves redistributed because of induced scattering off ions or electrons, $20-23$ ii) rfsonant waves driving parametric instabilities or beating with ion-acoustic waves, ${ }^{10,24-26}$ and iii) nonlinear Landau damping of resonant pairs of waves with plasma electrons. 11

Once accurate linear theory is obtained, estimates for allowed wave-wave and wave-particle interactions with the primary streaming instability spectrum can be deduced. For example, can two waves $\left(w_{1}, \bar{k}_{1}\right)$ and $\left(w_{2}, \bar{k}_{2}\right)$ that both belong to the primary spectrum satisfy the condition $w_{1}-w_{2}=\left(\bar{k}_{1}-\bar{k}_{2}\right) \cdot \bar{v}$, and how does the resonant velocity $\bar{v}$ depend on the external magnetic field strength? In addition, possible direct interaction between the primary spectrum and the return-current-driven spectrum can be estimated.

This analysis is intended to at least isolate allowed nonlinear mechanisms and to determine the characteristic wavelength/velocity space associated with each mechanism.

C. Task 3: Steady State Injection Simulations - Scattered Beam

It should be very difficult to execute one- and two-dimensional injection simulations for the kinetic interaction, including all possible nonlinear mechanisms. The problem is twofold. First, a large number of particles per cell will be necessary to reduce the noise level sufficiently. Second, many cells are required because of very different wavelengths associated with the primary spectrum, return-current spectrum, and possible nonlinear processes. However, with accurate linear cheory and nonlinear estimates, it appears possible to design and implement one-dimensional simulations that include both parametric and induced scattering effects for a beam-to-plasma density ratio $n_{b} / n_{p} \cong 10^{-1}$ to $10^{-2}$. However, it is only planned to look at these effects 
separately, with the main emphasis on obtaining the correct steady state quasilinear spectrum in two dimensions.

The code CCUBE contains extensive experimental diagnostics such as diamagnetic loops, local probes, caiorimeters, Faraday cups, and Rogowski loops. In addition, local measurements for the anomalous collision frequency are expected in both one and two dimensions. Comparison between simulated experimentai diagnostics and more exact code calculations will be done, for example, the inferred and calculated resistive $\int_{0}^{\tau} \mathrm{J} \cdot$ Edt heating. Also, the runs will provide initial insight into the dominant processes as well as provide the evolved distribution functions.

D. Task 4: Beam Self-Fields

To investigate the character of perpendicular velocity components caused by self-field effects (see Appendix), a number of simple planar diode simulations will be carried out using CCUBE. These time and space dependent calculations will be supplemented by steady state diode calculations supplied by PI. In addition, test particle orbit calculations will be carried out for given zero order self- and applied fields. From these calculations, the distribution of the velocity components at the anode foil entrance as a function of beam radius will be obtained, which can then be incorporated into the simulation code CCUBE to study self-field effects on adiabatic compression.

E. Task 5: Adiabatic Compressicn

Because of limitations associated with the diode, it is expected that some adiabatic compression of the beam will be necessary in the reactor regime. To obtain significant compression of intense beams, it is suggested (see Appendix) that the beam be first compressed in vacuum to reduce self-field effects followed by a second compression in plasma.

As a result, it is proposed to study adiabatic compression in both vacuum and plasma. The specific issue is whether the self-magnetic field effects, which limit compression in partially ionized gas, can be reduced significantly by performing the initial compression in vacuum. The initial distribution functions will be those obtained from previous diode calculations. Based upon these studies and PI models, ${ }^{36}$ realistic compression ratios and distribution functions will be estimated. 


\section{PROPOSED SECOND-YEAR PROGRAM}

During the second year, the emphasis will shift to the study of the nonlinear relaxation of the beam.

A. Task 1: Partial Numerical Simulation - Scattered Beam

Using the linear theory obtained from the previous year, multiwave partial numerical simulation codes will be implemented to study the quasi-linear relaxation of the beam, including oblique wave propagation. These calculations will be checked using the one- and two-dimensional codes REL, WAVE, and CCUBE. Expected results are the angle and energy spread of the evolved distribution function over a large parameter range. Based upon these calculations, a review of the analytic work will be undertaken to determine which calculations, if any, are essentially correct in the initial assumptions concerning the nonlinear steady state spectrum. Depending upon the results of this review, relevant analytical calculations will be done in conjunction with PI.

B. Task 2: Linear Theory and Nonlinear Estimates - Warm Beam

Based upon one- and two-dimensional code and partial numerical simulation results, the major changes with respect to energy and angular spread will be incorporated into a new class of distribution function. Appropriate linear theory associated with the new distribution will then be obtained. In addition, estimates for the characteristic wavelength/velocity space associated with allowed nonlinear processes will be carried out. The main aim here is to determine the differences in the spectrum caused by energy spread and angular spread. It is known, for example, that, for an unmagnetized beam-plasma interaction, the linear theories for a perfectly scattered beam (no energy spread) and a perfectly warm beam (no scatter) are quite different in character. ${ }^{2}$ C. Task 3: Steady State Injection Simulations - Scattered Beam

A series of two-dimensional steady state injection simulations will be carried out for varying initial beam angular scatter, for $n_{b} / n_{p} \cong 10^{-1}$ to $10^{-2}$. Also, the one-dimensional code REL will be used to study the steady state injection problem, the results of which will be compared with the two-dimensional runs. The particle simulation results, partial numerical simulation results, and analytical results obtained at both PI and LASL will then be compared with the PI experimental data to determine the major scaling characteristics of the heating. Isolated runs with a significant change in the magnetic field strength, for example, may be necessary, depending on the agreement between the theory and experiment. 


\section{Task 4: Net Current Code}

For direct experimental support, a hybrid code including ionization, recombination, and radiation (rate equations) together with a generalized ohm's law and field equation for the self-magnetic field will be developed. This type of code has been used previously for studying electron beam injection into dense gases (classical resistivity) for laser applications. ${ }^{37,38}$ In our applim cation, initial insight for the anomalous resistivity gained from the particle codes will be included to obtain the net current as a function of beam and plasma radial profiles, iadial boundary conditions, ionization state, and anomalous collision frequency. The calculated net current wili then be compared with the two-dimensional code results to provide a cons istent short-time calculation. Finally, the net current will be calculated on the beam time scale and compared with the PI experimental results.

\section{PRCPOSED THIRD-YEAR PROGRAM}

During the third year, the nonlinear spectrum characteristics of beams with combined angular and energy sprsad will be calculated. Also, stability of a cold beam and the effect of a longitudinal density gradient on the hydrodynamic interaction will be considered.

\section{A. Task 1: Parcial Numerical Simulation - Warm Beam}

Multiwave partial numerical simulation codes will be implemented to study the quasi-linear relaxation of a warm beam, including the oblique wave approximation. Again, the calculations will be checked using the one- and two-dimensional codes REL, WAVE, and CCUBE. Combining these results with the estimates for the allowed nonlinear interactions, it should be possible to determine if the dominant nonlinear mechanism found previously for a scattered beam is altered significantly when the distribution function is spread in energy as well as angle. Based upon previous calculations, the nonlinear spectrum should become more two-dimensional in character as the beam becomes spread in energy. ${ }^{2}$ Depending on the results of this anaiysis, it may be necessary to carry out additional analytical caiculations in conjunction with PI.

B. Task 2: Steady State Injection Simulations - Warm Beam

The code CCUBE will be used for two-dimensional steady state injection studies for the warm beam. Also, the one-dimensional code REL will be used to study the warm beam interaction. The aim is to again obtain insight into any 
major modifications in the anomalous resistivity and associated dominant nonlinear mechanisms caused by the presence of energy spread in the beam.

C. Task 3: Longitudinal Gradient Injection Simulations - Cold Beam

Many injection runs are planned to study the effect of a longitudinal ionization gradient on the hydrodynamic interaction. These runs include the finite bandwidth of the spectrum, and thus a more quantitative estimate for the reduction os the coupling efficiency in the presence of a density gradient is expected.

D. Task 4: Beam Stability [Filamentation and Flute $(\ell \neq 0)]$

An electromagnetic, coupled linear differential equation code will be developed and implemented to study beam stability (linear theory) with respect to filamentation and flute formation. The zero-order equilibrium will be assumed to be independent of the propagation direction but will allow arbitrary radial beam and plasma density profiles, self-magnetic and electric fields, and external magnetic field. The monoenergetic bean interaction will be considered. Initially, the plasma will be included in the cold fluid limit, but plasma temperature and diamagnetism may subsequently be incorporated into the code.

This finite boundary code can be used in conjunction with the net current code to obtain stability conditions for filamentation and fluting in the 1- to 3-m experiments and reactor regime. The numerical results will be used as a guide for the $P I$ analytical calculations. In addition, the effect of radiai density profiles and boundary conditions on the beam-plasma interaction will be estimated.

\section{REACTOR AND GENERAL APPLICATIONS}

The proposed theoretical program is directly applicable to the advanced concept electron beam-heated linear solenoidal reactor. As presently coriceived, such a reactor is 300-500 $\mathrm{m}$ long, with the intense electron beam coriverting its streaming energy into plasma thermal energy as it propagates along the solenoid. Because the energy transfer process is anomalous, the relativistic electron beam is an extremely versatile plasma heating source. In the hydrodynamic regime the deposition length is 2-100 cm with 40-70\% transfer efficiency, 19,27 whereas in the weak kinetic regime the deposition length has been estimated to be many orders of magnitude longer. ${ }^{20-26}$ Thus, the proposed program is also applicable to heating -ther advanced concept reactors, as well as main line fusion devices. In addition, the analysis has some bearing on 
beam interactions with blow-off plasmas for electron beam pellet fusion, the recent heavy-ion pellet fusion concept, and, to a lesser extent, the hot electron problem associated with the laser fusion concept.

On a more general level, the study of beam self-magnetic field effects, adiabatic compression, beam stability, and linear/nonlinear theory of a scattered relativistic beam propagating along a magnetic field is applicable to some collective ion acceleration schemes. ${ }^{39-41}$

\section{APPENDIX}

\section{BEAM SELF-FIEI.DS AND ADIABATIC COMPRESSION}

One problem in obtaining agreement between theory and experiment for the magnitude of heating is the presence of a radially dependent perpendicular velocity component $v_{1}$, generated by the discontinuity in the selfomagnetic fields of the beam between the diode and ciense plasma. Here $v_{1}$ refers to those components of velocity that are perpendicular to the external magnetic field. An approximate rule of thumb for the perpendicular component caused by the selfmagnetic field is that it increases linearly with beam radius,

$$
\frac{v}{c}=\frac{r}{a} \tan ^{-1}\left[\frac{B_{\theta}(r=a)}{B_{z}}\right] \text {, }
$$

with $B_{\theta}(r=a)$ being the self-magnetic field at the beam edge.

Recent two-dimensional cold beam injection simulations indicate ${ }^{19}$ that this component is a finite Larmor radius effect that can reduce the local beam coupling efficienc $y$ with increasing beam radius. For a scattered or warm beam interaction, the perpendicular velocity components associated with ine beam temperature may be larger than those resulting from the self-fields, in which case the coupling efficiency would be essentially independent of beam radius. This condition can be achieved if the diode generated beam interacts with the plasma directly. 
Because of Child-Langmuir limitations on diode current density and associated diode closure limitations on the pulse width, some adiabatic compression of the beam probably will be necessary before injection into the plasma in the reactor regime. Consequently, some compressed beam experiments are planned on the 1 - and 3-m target plasmas.

To avoid space charge limitations, beams are generally compressed in the presence of a partially ionized gas. ${ }^{36}$ For low compression ratios, most if an initially intense beam can be transmitted. Under these conditions, the distribution function is modified in a straightforward manner. As the compression ratio is increased, however, two limits on the transmission with resultant modifications in the distribution function show up.

At the anode foil, the perpendicular velocity components caused by the self-magnetic field increase, because the maximum magnetic field strength is determined by reactor considerations. Thus, electrons in the beam periphery will begin to reflect. For compression of mega ampere beams, Eq. (A-1) appears to 1 imit the compression ratio $M \cong 4$ for reactor size beams, neglecting anodefoil scattering.

For the compression to be adiabatic, the fractional change $f$ in the magnetic field strength over a Larmor orbit musi be small,

$$
\frac{\lambda_{c}(z)}{B_{z}(z)} \frac{d B_{z}(z)}{d z}=f<<1,
$$

where $\lambda_{c}(z)=2 \pi \gamma v_{11}(z) / w_{c}(z)$. Integrating Eq. (A-2) yields the length of the compression region

$$
\begin{aligned}
z_{c} & =\frac{\lambda_{c}^{\circ}}{f}\left[M \sqrt{1-\alpha^{2} / M}-\sqrt{1-\alpha^{2}}-\alpha^{2} \tanh ^{-1}\left(\sqrt{1-\alpha^{2} / M}\right)\right. \\
& \left.+\alpha^{2} \tanh ^{-1}\left(\sqrt{1-\alpha^{2}}\right)\right]
\end{aligned}
$$

as a function of the compression ratio $M$, final perpendicular velocity $a=v^{f} / c$, and $\lambda_{c}^{\circ}(\mathrm{cm}) \cong 10.6 \gamma / B_{f}(k G)$. In the weak magnetic field limit (at the entrance of the compression region), the nonlinear interaction length associated with the two-stream instability for a cold relativistic beam is 


$$
L_{N} \cong 5 \gamma \frac{n_{p}}{n_{b}}\left(\frac{1}{1+s^{2}}\right)^{1 / 3} \frac{c}{w_{p}},
$$

where $S=\beta_{0}^{2} \gamma\left(n_{b} / 2 n_{p}\right)^{1 / 3}$. The interaction length in Eq. $(A-4)$ is relatively independent of foil scattering provided that.

$$
\frac{\Delta n}{n_{b}}=1.0-\exp \left[\left(n_{b} / 2 n_{p}\right)^{1 / 3} / \gamma \theta_{s}^{2}\right] \geqq 0.15,
$$

where $\theta_{S}$ is the mean scattering angle. Comparing Eqs. $(A-3)$ and $(A-4), L_{N} \ll$ $z_{c}$ in the high-voltage and large-compression regimes. Thus, the transmission of the center of the beam is ultimately limited by collective effects.

To avoid the self-magnetic field (adiabatic change in the beam selfmagnetic field) and collective limirations on compression, one might consider compressing annular beams in vacuum. The space charge limit for a relativistic electron beam is 21

$$
I(k A)=\frac{17\left(\gamma^{2 / 3}-1\right)^{3 / 2}}{f(b)+2 \ln (R / a)},
$$

where $R$ is the wall radius, $a$ is the outer beam radius, and $b$ is the irner bean radius. For a thin annular beam where $a \cong b, f(b) \cong 0$, and in principle $a$ large current can be passed by allowing the beam to propagate sufficiently close to the wall,

$$
I(K A) \cong 8.5\left(\gamma^{2 / 3}-1\right)^{3 / 2} R / \mathrm{rB},
$$

where $r_{b}^{o}$ is the beam Larmor radius. However, compression of such a beam leads to the condition that $f(b) \gg 2 \ln (R / a)$, with the space charge limiting current given by

$$
I(k A) \cong \frac{17\left(\gamma^{2 / 3}-1\right)^{3 / 2}}{f(b)} \cong 300-400 k A
$$

for 10-MeV electrons. 
It has been suggested that the space charge limited current could be increased by decreasing the potential on axis to zero. For an annular bean this might be achieved by running wires along the compression axis 30 or by carrying out the compression in concentric cones. However, this technique might also provide a return-current path, thus producing perpendicular motion caused by seif-magnetic fieid discontinuity.

Taking all these considerations into account, it may be possible to obtain significant compression if an initially intense relativistic beam is first compressed in vacuum to reduce self-magnetic field effects then compressed in

plasma to overcome the space charge limitation. Because the perpendicular velocity components caused by foil scattering are increased during the vacuum compression phase, the strength of the collective processes is decreased. This effect, combined with the shorting of the plasma compression region, should, to a great extent, overcome limitations in transmission caused by collective effects.

\section{REFERENCES}

1. Physics International Company Proposal PIP-1770 (1976), "The Electron Beam Heated Solenoid Reactor: A Proof of Principle Experiment," Vol. I.

2. L. E. Thode, "Plasma Heating by Scattered Relativistic Electron Beams: Correlations Among Experiment, Simulation, and Theory," Phys. Fluids 19, 831 (1976); see references for preionized target gas experiments before 1975.

3. D. Prono, B. Ecker, N. Bergstrom, and J. Benford, "Plasma-Return-Current Heating by Relativistic Electron Beams with $v / y \sim 10$," Phys. Rev. Lett. 35, 438 (1975).

4. R. Okamura, Y. Nakamura, and N. Kawashima, "Microwave Emission from the Magnetized Plasma Heated by a Short Pulse Width Relativistic Electron Beam," Institute of Space and Aeronautical Science Research note 2 (1975), University of Tokyo, Meguro, Tokyo.

5. A. K. L. Dymoke-Bradshaw, A. E. Dangor, and J. D. Kilkenny, "Plasma Heating by an Intense Relativistic Electron Beam;" 6th International Conf. on Pilas. Phys. and Controlled Nucl. Fusion Research (Berchtesgaden, 1976), paper G2-4.

6. B. Jurgens, H. J. Hopman, P. de Haan, P. C. de Jagher, and J. Kistemaker, "Energy Transfer of a Relativistic Electron Beam to a Plasma," 6th International Conf. on Plas. Phys. and Controlled Nucl. Fusion Research (Berchtesgaden, 1976), paper G2-1. 
7. P. Sunka, K. Jungwirth, I. Kovac, V. Piffl, J. Stockel, and J. Ullschmeid, "Injection of $a$ Relativistic Electron Beam into an Inhomogeneous Magnetized Plasma," 6th International Conf. on Plasma Dhys. and Controlled Nucl. Fusion Research (Berchtesgaden, 1976), paper 62-2.

8. R. V. E. Lovelace and R. N. Sudan, "Plasma Heating by High-Current Reilativistic Electron Beams," Phys. Rev. Lett. 27, 1256 (1971).

9. N. Albright, "A Model of the Acoustic. Two-Stream Instability," Physics International Company report PIFR-6-75 (1975), San Leandro, California.

10. L. E. Thode and R. N. Sudan, "Plasma Heating by Relativistic Electron Beams: I. Two-Stream Instability," Phys. Fluids 18, 1552 (1975).

11. L. E. Thode and B. B. Godfrey, "Energy Lost by a Relativistic Electron Beam Propagating Along an External Magnetic Field," Phys. Fluids 19, 316 (1976).

12. R. I. Kovtun and A. A. Rukhadze, "Nonlinear Interaction of a Low-Density Relativistic Electron with a Plasma," Zh. Eksp. Teor. Fiz. 58, 1709 (1970) [Sov. Phys. -JETP 31, 915 (1970)].

13. A. Toepfer and J. Poukey, "Nonlinear Saturation of the Reiativistic BeamPlasma Instability in the Presence of Ion Density Fluctuations," Phys. Fluids 16, 1546 (1973).

14. G. Benford, "Radiation and Ionization of Relativistic Electron Beams," Plasma Phys. 10, 203 (1973).

15. M. Lampe and P. Sprargle, "Saturation of the Relativistic Two"Stream Instability by Electron Trapping," Phys. Fluids 18, 475 (1975).

16. N. G. Matsiborko, I. N. Onishchenko, V. D. Shapiro, and V. I. Schevchenko, "On Nonlinear Theory of Instability of a Monoenergetic Electron Beam in a Plasma," Plasma Phys. 14, 591 (1972).

17. Ya. B. Fainberg, Y. D. Shapiro, and V. I. Shevchenko, "Nonl inear Theory of Interaction Between a Mono-Chromatic Beam of Reiativistic Electrons and a Plasma," Zh. Eksp. Teor. Fiz. 57, 966 (1969) [Sov. Phys. -JETP 30, 528 (1970)].

18. R. L. Ferch and R. N. Sudan, "Linear Two-Stream Instability of Warm Relativistic Electron Beams," Plasma Phys. 17, 905 (1975).

19. L. E. Thode, "The Role of Collisions and Finite Boundary Conditions on the Beam-Plasma Interaction," Bul1. Am. Phys. Soc. 21, 1077 (1976).

20. B. N. Briezman and S. G. Feizov, "The Helicon Mechanism of Reiaxation of a Relativistic Electron Beam in a Plasma," Zh. Eksp. Teor. Fiz. 66, 200 (1974) [Sov. Phys. -JETP $\underline{33}, 93$ (1974)].

21. B. N. Breizman and D. D. Ryutov, "Powerful Relativistic Electron Beams in a Plasma and in Vacuum (Theory)," Nucl. Fusion 14, 873 (1974). 
22. L. I. Rudakov, "Collective Slowing Down of an Iritense Beam of Relativistic Electrons in a Dense Plasma Target," Zh. Eksp. Teor. Fiz. 59, 2091 (1970) [Sov. Phys. -JETP $\underline{32}, 1134$ (1971)].

23. A. T. Altyntsev, B. N. Briezman, A. G. Eskov, O. A. Zolotovsky, V. I. Koroteev, P. Kh. Kurtmullev, V. L. Maslov, D. D. Ryutov, and V. N. Semenov, "Collisioniess Relaxation of an Ultra-Relativistic Electron Beam in a Plasma," Nucl. Fusion Supp. 162 (1972).

24. T. S. T. Young, "Interactions Between High and Low Frequency Modes in Electron Beam-Plasma System," submitted to Phys. Fluids.

25. K. Papadopoulos, "Nonlinear Stabilization of Beam Plasma Interactions by Parametric Effects," Phys. Fiuids 18, 1769 (1975).

26. H. Shame1, Y. C. Lee, and G. J. Morales, "Parametric Excitation of Iun Density Fluctuations in the Relativistic Beam-Plasma Interaction," Phys. Fluids 19, 849 (1976).

27. L. E. Thode, "Plasma Heating by Relativistic Electron Beams: Experiment, Simulation, and Theory," Bul1. Am. Phys. Soc. 21, 532 (1976).

28. M. B. Levin, M. G. Lynbarskii, I. N. Onishchenku, V. D. Shapiro, and V. I. Shevchenko, "Contribution to the Nonlinear Theory of Kinetic Instability of an Electron Beam in Plasma," Zh. Eksp. Teor. Fiz. 62, 725 (1972) [Sov. Phys. - JETP 35, 898 (1972)].

29. A. A. Ivanov, V. V. Parail, and T. K. Soboleva, "Nonlinear Theory of the Interaction Between a Monoenergetic Beam and a Dense Plasma," Zh. Eksp. Teor. Fiz. 63, 1678 (1972) [Sov. Phys. -JETP 36,887 (1973)].

30. B. B. Godfrey, "Numerical Cherenkov Instabilities in Electromagnetic Particle Codes," J. Comput. Phys. 15,504 (1974).

31. C. W. Nielson and E. L. Lindman, "An Implicit, Two-Dimensional, Electromagnetic Plasma Simulation Code," in Proceedings of 6 th Conference on Numerical Simulations of Plasmas, Lawrence Berkeley Laboratory Conferences Report 73-0710 (Lawrence Berkeley Laboratory, Berkeley, California, 1973), paper E-3.

32. The new code was designed by B. B. Godfrey and is especially suited for relativistic electron beam problems, see the "Theoretical Division Annual Report - FY76," LA-6816-PR (1976), p. 148.

33. The diagnostic routines were designed by $L$. $E$. Thode: Agreement between code and experimental diagnostics was better than $20 \%$ in a recent study of space charge limited flow experiment of Y. I. Abrashitov., V. S. Koedan, V. V. Konyukhov, V. M. Lagunov, V. N. Luk'yanov, K. I. Mekler, and D. D. Ryutov, "Interaction of a High-Intensity Relativistic Electron Beam with Plasma in a Magnetic Field," Zh. Eksp. Teor. Fiz. 66, 1324 (1974) [Sov. Phys. -JETP 39, 647 (1974)]. 
34. B. B. Godfrey, W. R. Shanahan, and L. E. Thode, "Linear Theory of a Cold Relativistic Beam Propagating Along an External Magnetic Field," "hys. Fluids 18,346 (1975).

35. L. E. Thode, "Relativistic Streaming Instability Enhanced by Judicious Choice of External Magnetic Field," in the Conference Record of the 1978 IEEE International Conference on Plasma Science (Monterey, Callifornia, 1978), P. 284.

36. T. S. T. Young and P. Spence, "Model of Magnetic Compression of Relativistic Electron Beams," Appl. Phys. Lett. 29, 464 (1976); this paper gives a short review of adiabatic compiession experiments.

37. J. W. Poukey and A. J. Toepfer, "Comments on Reverse Current Induced by Injection of a Relativistic Electron Beam into a Pinched Plasma," Phys. Fluids 17, 505 (1974).

38. D. A. McArther and J. W. Polikey, "Plasma Created in a Neutral Gas by a Relativistic Electron Beam," Phys. Fluids 16, 1996 (1973).

39. M. L. Sloan and W. E. Drummond, "Autoresonant Accelerator Concept:," Phys. Rev. Lett. 31, 1234 (1973).

40. P. Sprangle, A. T. Drobot, and W. M. Manheimer, "Collective Ion Acceleration in a Converging Waveguide," Phys. Rev. Lett. 36, 1180 (1976).

41. R. J. Faehl, R. B. Miller, and B. B. Godfrey, "Simulation of Virtual Cathode Formation During Vacuum Propagation," Bu11. Am. Phys. Soc. 21, 1165 (1976). 